\title{
A 3D lattice Boltzmann effective media study: understanding the role of C-S-H and water saturation on the permeability of cement paste
}

\author{
M Zalzale ${ }^{1}$, P J McDonald ${ }^{2}$ and K L Scrivener ${ }^{1}$ \\ ${ }^{1}$ Laboratory of Construction Materials, Ecole Polytechnique Fédérale de Lausanne (EPFL), station 12, \\ CH-1015 Lausanne, Switzerland \\ ${ }^{2}$ Department of Physics, University of Surrey, Guildford, Surrey, GU2 7XH, UK \\ Email: p.mcdonald@surrey.ac.uk
}

\begin{abstract}
A 3D lattice Boltzmann model is developed and used to calculate the water and gas permeabilities of model cement pastes at different degrees of water saturation. In addition to permeable micron-sized capillary pores and impermeable solid inclusions, the lattice Boltzmann model comprises weakly-permeable nano-porous calcium silicate hydrate (C-S-H). The multi-scale problem is addressed by using an effective media approach based on the idea of partial bounce-back. The model cement paste microstructures are generated with the platform $\mu$ ic. The critical parameters, C-S-H density and capillary porosity, are taken from ${ }^{1} \mathrm{H}$ nuclear magnetic resonance relaxation analysis. The distribution of water and air is defined according to the Kelvin-Laplace law. It is found that when the capillary porosity is completely saturated with a fluid (either water or gas), the calculated intrinsic permeability is in good agreement with measurements of gas permeability on dried samples $\left(10^{-17}-10^{-16} \mathrm{~m}^{2}\right)$. However, as the water saturation is reduced, the calculated apparent water permeability decreases and spans the full range of experimentally measured values $\left(10^{-16}-10^{-22} \mathrm{~m}^{2}\right)$. It is concluded that the degree of capillary water saturation is the major cause for variation in experimental permeability measurements. It is further concluded that the role of the weaklypermeable C-S-H, omitted in earlier modelling studies, is critical for determining the permeability at low capillary saturation.
\end{abstract}

\section{Keywords}


Permeability; Cement; Lattice Boltzmann; Effective Media; Partial Bounce-back; Darcy-Brinkman;

\section{PACS numbers}

47.56. $+\mathrm{r} \quad$ Flows through porous media

91.60.Np Permeability and porosity

\section{Introduction}

Concrete is the most widely used construction material on Earth with an estimated production of over one cubic meter per person per year [1]. Although it is a material with low specific $\mathrm{CO}_{2}$ emissions, the huge volumes mean that concrete is responsible for some 5 to $8 \%$ of global $\mathrm{CO}_{2}$ emissions [2]. It is consequently of great environmental and economic interest to design new concretes with improved and predictable resistance to degradation. Cement paste is the continuous porous matrix that binds aggregates (sand and stones) to form concrete. It constitutes around $30 \%$ of concrete volume and has a critical role in determining its porosity and permeability. Since water ingress or egress is implicated in almost every form of concrete deterioration, including corrosion of steel reinforcement and freeze-thaw attack, porosity and permeability measurements are often used as indicators of durability.

Porosity is the fraction of a material that is not solid. In cement pastes, pores sizes range from nanometre spaces within the calcium silicate hydrate (C-S-H) to micron-sized capillary pores. Depending on the internal relative humidity, pores may be filled with water or air. According to the Kelvin-Laplace law, as the relative humidity decreases during drying, large pores will empty before small pores.

The intrinsic permeability $\kappa$, which may be expressed in units of $\mathrm{m}^{2}$, characterises the medium from the perspective of pressure-induced fluid flow through the fully saturated porosity. Theoretically, it is a property of the medium and is independent of the penetrating fluid ${ }^{3}$ and

\footnotetext{
${ }^{3}$ The non-intrinsic permeability, also called hydraulic conductivity, is fluid dependent and is expressed in $\mathrm{m} / \mathrm{s}$. The ratio of intrinsic permeability to hydraulic conductivity is given by $\mu / \rho g$
} 
applied pressure. It is defined in terms of macroscopic observables through Darcy's law [3] as:

$$
\kappa=\frac{L}{A} \frac{J \mu}{\Delta P}
$$

where $L$ and $A$ are the length and cross sectional area of a test sample through which a fluid flow $J$ is driven by an external pressure difference $\Delta P$. The dynamic fluid viscosity $\mu$ is the product of the density $\rho$ and the kinematic viscosity $v$.

The intrinsic permeability of cement paste is challenging to measure with water as it is very difficult to force a measurable liquid flow through the paste. Measurement of gas permeability is easier, but requires prior drying of the sample to a well-defined state of saturation, which may take more than one year. Hence, indirect experimental methods such as beam-bending [4] have also been employed. The intrinsic permeability values reported in literature vary widely from circa $10^{-22}$ to $10^{-16}$ $\mathrm{m}^{2}$ as shown in table I for cement pastes with water-to-cement $(w / c)$ ratios by mass ranging from 0.35 to 0.47 . Contrary to expectations, the measured values reported suggest that the intrinsic permeability of cement paste is strongly dependent on the penetrating fluid. Measurements with oxygen as the penetrating fluid are $3-5$ orders of magnitude greater than typical values obtained using water. A similar discrepancy is widely observed for other cement-based materials [5, 6]. Moreover, for the same fluid - water - the permeability measurements span $2-3$ orders of magnitude. Even for nominally similar materials with comparable cement composition, w/c ratio and hydration age, the measured permeability values differ by a factor of 4 [7].

Table I: Experimental permeability measurements for mature cement paste with water-tocement mass ratios $(w / c)$ ranging from 0.35 to 0.47 .

\begin{tabular}{|c|c|c|c|c|c|c|}
\hline & Method & Fluid & Conditioning & $w / c$ & $\begin{array}{l}\text { Age } \\
\text { (days) }\end{array}$ & $\begin{array}{l}\text { Intrinsic } \\
\text { permeability } \\
\left(\mathrm{m}^{2}\right)\end{array}$ \\
\hline $\begin{array}{l}\text { Nyame \& } \\
\text { Illston } 1981 \\
{[8]}\end{array}$ & $\begin{array}{l}\text { Standard } \\
\text { permeability } \\
\text { cell }\end{array}$ & Water & $\begin{array}{l}\text { Cured underwater (no } \\
\text { information provided } \\
\text { about the delay before } \\
\text { placing underwater) }\end{array}$ & 0.47 & 600 & $5 \times 10^{-22}$ \\
\hline $\begin{array}{l}\text { Banthia and } \\
\text { Mindess }\end{array}$ & $\begin{array}{l}\text { Tri-axial } \\
\text { permeability }\end{array}$ & Water & Sealed for $24 \mathrm{~h}$ then & 0.35 & 28 & $8.9 \times 10^{-20}$ \\
\hline
\end{tabular}

where $\rho$ is the fluid density, and $g$ is the acceleration due to gravity. For water at $20^{\circ} \mathrm{C}$, $\mu / \rho g \approx 10^{-7}$ m.s. 


\begin{tabular}{|c|c|c|c|c|c|c|}
\hline 1989 [9] & cell & & cured underwater & & & \\
\hline $\begin{array}{l}\text { Ai et al. } \\
2001[10]\end{array}$ & $\begin{array}{l}\text { Thermo- } \\
\text { permeametry }\end{array}$ & Water & $\begin{array}{l}\text { Sealed and moist } \\
\text { cured for } 24 \mathrm{~h} \text { then } \\
\text { cured underwater }\end{array}$ & 0.4 & 548 & $10^{-22}$ \\
\hline $\begin{array}{l}\text { Vichit- } \\
\text { Vadakan \& } \\
\text { Scherer } \\
2002[4,7]\end{array}$ & $\begin{array}{l}\text { Beam- } \\
\text { bending }\end{array}$ & Water & $\begin{array}{l}\text { Sealed for } 48 \mathrm{~h} \text { then } \\
\text { cured underwater }\end{array}$ & 0.4 & 14 & $5 \times 10^{-22}-10^{-21}$ \\
\hline $\begin{array}{l}\text { Vichit- } \\
\text { Vadakan \& } \\
\text { Scherer } \\
2003 \text { [11] }\end{array}$ & $\begin{array}{l}\text { Beam- } \\
\text { bending }\end{array}$ & Water & $\begin{array}{l}\text { Sealed for } 18 \mathrm{~h} \text { then } \\
\text { cured underwater }\end{array}$ & 0.45 & 3 & $6 \times 10^{-22}$ \\
\hline $\begin{array}{l}\text { Ye } 2005 \\
{[12]}\end{array}$ & $\begin{array}{l}\text { Three parallel } \\
\text { permeability } \\
\text { cells }\end{array}$ & Water & $\begin{array}{l}\text { Sealed. Vacuum } \\
\text { saturated with water } \\
\text { for 4-8h prior to } \\
\text { measurement }\end{array}$ & 0.4 & 28 & $9 \times 10^{-21}$ \\
\hline $\begin{array}{l}\text { Grasley \& } \\
\text { Scherer } \\
2007 \text { [7] }\end{array}$ & $\begin{array}{l}\text { Dynamic } \\
\text { pressurization }\end{array}$ & Water & $\begin{array}{l}\text { Sealed for } 12-18 \mathrm{~h} \text { then } \\
\text { cured underwater }\end{array}$ & 0.4 & 14 & $2 \times 10^{-21}$ \\
\hline $\begin{array}{l}\text { Wong et al. } \\
2009[13]\end{array}$ & $\begin{array}{l}\text { Standard } \\
\text { permeability } \\
\text { cell }\end{array}$ & Oxygen & $\begin{array}{l}\text { Sealed. Dried prior to } \\
\text { measurement }\end{array}$ & 0.45 & 90 & $6.5 \times 10^{-17}$ \\
\hline
\end{tabular}

Several hypotheses have been advanced to try and explain the broad range of measured permeability values in cement-based materials including: gas slippage on the surface of the pores [5]; microcracks induced by sample drying prior to gas permeability measurements [13]; delayed cement hydration [6, 14]; swelling of cement hydrates in contact with water [14] and dissolution and migration of fine elements $[6,9,14,15]$. However, careful examination shows that none of these is sufficient to explain fully the wide variation of results. A more plausible hypothesis is variation in the degree of water saturation, defined as the fraction of the porosity that is filled with water [14, 16-18]. In particular, Abbas et al. [16], Baroghel-Bouny et al., [18] and Wong et al. [17] showed a strong dependency of the intrinsic gas permeability on the degree of water saturation for concretes, mortars and other cement-based materials. However, we are not aware of any similar studies on cement paste using water as the permeating fluid. Although experimentalists try to ensure water saturation before measuring water permeability, total saturation is very difficult to establish and maintain. Careful analysis of the procedures leading to the results reported in table I, for reasons outlined in the next 
section, leads us to believe that none of the measurements of water permeability to date has actually been carried out on a fully saturated material.

Hence, in this paper, we seek to demonstrate the effect of partial water saturation on the apparent intrinsic permeability of cement paste. Due to the inherent difficulties of sample preparation and / or experimental method, we choose to do this by modelling fluid flow through cement paste model microstructures. Lattice Boltzmann methods are chosen because they deal implicitly with arbitrarily shaped geometries and because the algorithms are highly amenable to parallelization.

This is not the first time that lattice Boltzmann methods have been applied to calculate the permeability of cement paste: Garboczi and Bentz [19], Zalzale and McDonald [20] and Zhang et al. [21] have made earlier studies. In general they find that for a mature cement paste with a water-tocement ratio of 0.4 , the intrinsic permeability is of the order of $10^{-18} \mathrm{~m}^{2}$, several orders of magnitude larger than the lowest water permeability measurement and closer to the gas result. However, these previous models have significant limitations. First, previous simulations [19-21] only addressed fully saturated porosity and cannot be compared to water based experiments with saturation less than $100 \%$. Second, as the cement powder reacts with water, the micron-sized capillary porosity fills with nano-porous hydration products, principally C-S-H. As the reaction proceeds, the water-filled capillary porosity depercolates and the nano-porosity of the C-S-H becomes crucial to maintain the percolation of the water-filled pore network. Previous simulations only addressed the capillary porosity and ignored the nano-scale porosity. Treating the C-S-H phase as impermeable solid with no inherent porosity makes the percolation of the pore network, and thus the permeability, critically dependent on the fineness of the resolution of the microstructures $[19,20]$. These factors lead to an overestimation of the permeability at large porosity and cause it to fall catastrophically to zero below the percolation threshold of the capillary porosity. We also note that in previous work, the simulation results were compared to a limited set of experimental measurements and did not discuss the conditioning of the samples or the large scatter as shown in Table I.

Due to the limitation of computing resources, it is impossible to describe structures with features ranging over 3 - 4 orders of magnitude in size within a conventional discrete LB 
implementation. To overcome this difficulty, we adopt LB effective media methods based on the ideas of partial bounce-back [22-24] to describe the C-S-H phase. Hence, each numerical node represents a permeable capillary pore, or an element of weakly-permeable nano-porous C-S-H, or an impermeable solid inclusion such as unhydrated cement. Effective media approaches are preferred to multi-scale grids and memory-efficient implementations because they do not require a detailed knowledge of the structure of the nano-porous C-S-H that is still an area of active debate [25-27].

In summary, the novelty of this work is:

- The inclusion of the C-S-H nano-porosity in simulations of cement paste permeability. This allows us to maintain the percolation of the pore network at computationally accessible, moderate resolutions. Consequently, we can calculate a permeability even when the network of capillary pores is depercolated. Furthermore, the inclusion of the C-S-H as an effective medium with a prescribed permeability allows us to quantify the effect of the permeability of the C-S-H on the permeability of the cement paste.

- The calculation of the apparent intrinsic permeability of cement paste at partial water saturation including a study of the differences attributable to liquid or gas measurements.

- A comparison of numerical results with a large set of experimental permeability measurements and a plausible explanation of the effect of sample preparation on measured permeability.

We believe that this is the first time that LB partial bounce-back methods have been applied to a complex 3D microstructure. We show that as the water saturation is reduced, the calculated apparent intrinsic permeability spans the full range of experimentally measured values $\left(10^{-16}\right.$ to $10^{-22}$ $\mathrm{m}^{2}$ ). We argue that the results go a long way to explaining the very wide range of experimental data in literature as well as a considerable part of the difference between intrinsic permeability as measured with water and gas.

\section{Cement paste: porosity and saturation}


Cement paste is made by mixing anhydrous cement powder with water. The chemical reactions between the anhydrous cement and water lead to the formation of hydrates, which fill the original space to produce a porous solid. The main hydrate phase, calcium silicate hydrate (C-S-H), is a nano-porous material comprising inter-layer spaces $(\approx 1 \mathrm{~nm})$ and gel pores $(3-5 \mathrm{~nm})$ [27]. The space not filled by hydrates is referred to as capillary porosity. The absolute volume of the solid plus liquid part of the cement paste decreases with time because the volume of the hydrates is smaller than the combined volumes of the reactants (anhydrous cement and water). This is called chemical shrinkage. Initially this decrease is accommodated by overall changes in the volume of the paste. However, from initial setting, the paste starts to resist deformation which causes the formation of micron-sized voids within the capillary porosity. Without an external source of liquid water, these pores remain empty. Unreacted water occupies other capillary pores with sizes ranging up to a few microns [27]. The total capillary porosity is of the order of $7-11 \%$ in a typical well reacted paste. One therefore expects the capillary porosity to be percolated. However, as little as one tenth of this volume may actually be water-filled, as demonstrated in recent quantitative nuclear magnetic resonance (NMR) experiments [27]. Hence, the water-filled porosity may not be percolated.

The differences in water saturation of the capillary porosity primarily originate from the conditioning of the samples after mixing. In systems which are cured sealed, the chemical shrinkage voids are necessarily devoid of liquid water. In underwater cured systems, it is often considered that water is drawn into the voids as they form. However, this very much depends on the size of the sample and the delay in exposing the paste to additional water. Water cannot permeate across more than millimetre-sized samples on the timescale of the curing due to the rapidly decreasing permeability of the paste. Hence for large samples, such as might be used for permeability tests, and / or for water exposure delays of more than a few hours, a significant fraction of the chemical shrinkage porosity can remain devoid of water. This is confirmed by recent NMR experiments, which have shown that it is difficult, if not impossible, to subsequently saturate more than about half of the voidage even using vacuum saturation on small samples [28].

\section{Lattice Boltzmann methods}




\subsection{The standard lattice Boltzmann method}

In lattice Boltzmann methods, at each node of a discrete lattice mesh, the fluid is represented by a density distribution of $Q$ fluid elements each with a defined lattice velocity performing consecutive propagation and collision steps. The standard LB method is encapsulated in:

$$
f_{i}\left(\boldsymbol{r}+\boldsymbol{e}_{i} \Delta t, t+\Delta t\right)-f_{i}(\boldsymbol{r}, t)=\Delta t \sum_{j=1}^{Q} S_{i j}\left(f_{j}(\boldsymbol{r}, t)-f_{j}^{e q}(\boldsymbol{r}, t)\right) .
$$

Here $\boldsymbol{r}$ is a lattice node, $\boldsymbol{e}_{i}$ are the allowed velocities of the fluid elements with $i=1 \ldots Q$, and $\Delta t$ is the lattice time step. The functions $f_{i}$ represent the density of fluid moving at position $\boldsymbol{r}$ and time $t$ with velocity $\boldsymbol{e}_{i}$. The term on the right hand side of equation (2) is the collision operator in which $S_{i j}$ is the collision matrix that serves to relax the fluid towards an equilibrium distribution $f_{j}^{e q}(\boldsymbol{r}, t)$ that encapsulates the physics of the problem. The macroscopic density $\rho(\boldsymbol{r}, t)$ and momentum $\rho(\boldsymbol{r}, t) \boldsymbol{u}(\boldsymbol{r}, t)$ are calculated from the distribution functions as:

$$
\begin{gathered}
\rho(\boldsymbol{r}, t)=\sum_{i=1}^{Q} f_{i}(\boldsymbol{r}, t), \\
\rho(\boldsymbol{r}, t) \boldsymbol{u}(\boldsymbol{r}, t)=\sum_{i=1}^{Q} f_{i}(\boldsymbol{r}, t) \boldsymbol{e}_{i} .
\end{gathered}
$$

To recover the incompressible Navier-Stokes equation for fluid motion, the equilibrium distribution functions are [29]:

$$
f_{i}^{e q}(\boldsymbol{r}, t)=\omega_{i} \rho\left(1+\frac{\boldsymbol{u}^{*} \cdot \boldsymbol{e}_{i}}{c_{s}^{2}}+\frac{\left(\boldsymbol{u}^{*} \cdot \boldsymbol{e}_{i}\right)^{2}}{2 c_{s}^{4}}-\frac{\boldsymbol{u}^{*} \cdot \mathbf{u}^{*}}{2 c_{s}^{2}}\right)
$$

where the constants $\omega_{i}$ are lattice weights specific to the chosen lattice, $\boldsymbol{u}^{*}$ is the equilibrium microscopic velocity equal to the macroscopic velocity $\boldsymbol{u}$ in absence of external forcing, $c_{s}=c / \sqrt{3}$ is the lattice speed of sound with $c=\Delta x / \Delta t$ the lattice speed and $\Delta x$ the lattice spacing. 
The collision matrix can be simplified by using a single-relaxation-time collision operator [29]

$S_{i j}=-\frac{1}{\tau} \delta_{i j}$ where the relaxation time $\tau$ is related to the kinematic fluid viscosity $v$ through

$v=c_{s}^{2}(\tau-\Delta t / 2)$ and $\delta_{i j}=\left\{\begin{array}{l}0 \text { if } i \neq j \\ 1 \text { if } i=j\end{array}\right.$ is the Kronecker delta. Setting $\Delta x=\Delta t=\tau=1$, equation

reduces to:

$$
f_{i}\left(\boldsymbol{r}+\boldsymbol{e}_{i}, t+1\right)=f_{i}^{e q}(\boldsymbol{r}, t)=\omega_{i} \rho\left(1+3 \boldsymbol{u}^{*} \cdot \boldsymbol{e}_{i}+\frac{9}{2}\left(\boldsymbol{u}^{*} \cdot \boldsymbol{e}_{i}\right)^{2}-\frac{3}{2} \boldsymbol{u}^{*} \cdot \boldsymbol{u}^{*}\right)
$$

One of the major advantages of LB methods is the ability to handle highly irregular boundaries by means of simple arithmetical operations. At fluid-solid interfaces, the no-slip boundary condition [30] is often approximated with the bounce-back condition: if a fluid element hits a solid boundary following the propagation step, its momentum is reversed so that:

$$
f_{i}\left(\boldsymbol{r}+\boldsymbol{e}_{i}, t+1\right)=f_{\tilde{i}}(\boldsymbol{r}, t)
$$

where $\tilde{i}$ is the direction opposite to $i: \boldsymbol{e}_{i}=-\boldsymbol{e}_{\tilde{i}}$.

\subsection{Lattice Boltzmann methods with effective media}

In effective media approaches, the multi-scale problem is addressed by assigning effective macro-scale properties (i.e. permeability) to appropriate numerical nodes. These approaches are capable of extending the use of standard numerical methods to include larger media or media with partly unknown geometry. There are two classes of lattice Boltzmann solvers that incorporate effective media: force-adjusted and partial bounce-back.

The force-adjusted method has its origins in the work of Spaid and Phelan [31] and was further developed by Freed [32] and Martys [33]. The principle of the method is to adjust the velocity term in the LB equilibrium functions in order to reduce the magnitude of the momentum. By doing so, the Stokes equation is used to model the flow in open regions, and the Brinkman equation is used to model the flow through porous structures by treating them as an effective medium of prescribed permeability. This method was applied to study the effect of sand grain permeability on the bulk permeability of Fontainebleau sandstone in three dimensions by Martys and Hagedorn [34]. The 
primary limitation is an inability to model systems with very low permeability, less than order of $10^{-13}$ $\mathrm{m}^{2}$ [34]. The method was therefore judged not suitable for cement paste for which $\kappa_{C-S-H} \approx 10^{-22} \mathrm{~m}^{2}$. The partial bounce-back method was proposed by Dardis and McCloskey [22, 35] based on earlier work on lattice gases by Balasubramanian et al. [36] and Gao and Sharma [37]. In this method, the fluid collision and bounce-back rules are combined to reflect local material properties (Figure 1). Variations of this method have been developed by Thorne and Sukop [23, 38] and Walsh et al. [24]. Walsh et al. showed that the three algorithms [22-24] produce equivalent results although their own has significant advantages in terms of mass conservation in heterogeneous media and suitability for parallel computing.

To control the partial bounce-back step, an effective media parameter $\sigma(\boldsymbol{r})$ is introduced ${ }^{4}$ [24]:

$$
f_{i}\left(\boldsymbol{r}+\boldsymbol{e}_{i}, t+1\right)=(1-\sigma(\boldsymbol{r})) f_{i}^{e q}(\boldsymbol{r}, t)+\sigma(\boldsymbol{r}) f_{\tilde{i}}(\boldsymbol{r}, t) .
$$

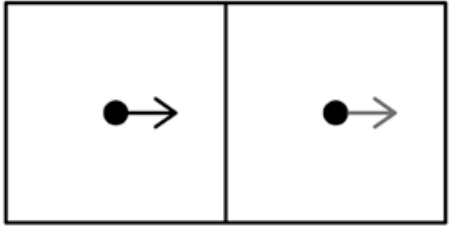

(a)

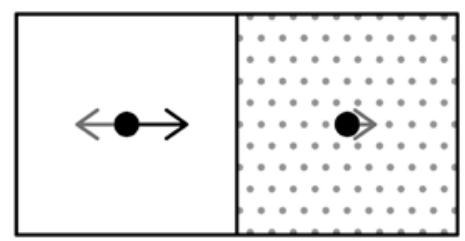

(c)

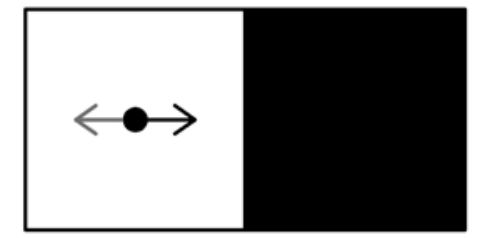

(b)

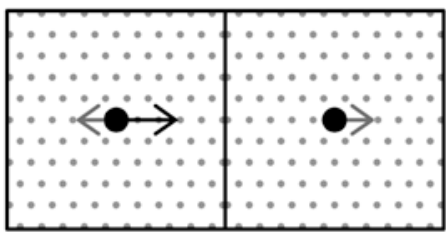

(d)

Figure 1. A 1D schematic showing one distribution function for (a) propagation between fluid nodes, (b) full bounce-back at a fluid-solid interface, (c) propagation and partial bounce-back between a fluid and a weakly-permeable node and (d) propagation and partial bounce-back between two weakly-permeable nodes. Pores are shown in white, solids in black and weakly-

\footnotetext{
${ }^{4}$ The "effective media parameter" $\sigma$ is equivalent to the "density of solid scatterers" or "solid fraction" previously used by other authors. We prefer the term "effective media parameter" to avoid unnecessary confusion with the inherent porosity.
} 


\section{permeable solids in dotted grey. Black arrows stand for the distribution function at time $t$ and} grey arrows for the distribution function at time $t+1$.

The microscopic and macroscopic density and momentum are given by:

$$
\begin{gathered}
\rho^{*}(\boldsymbol{r}, t)=\sum_{i=1}^{Q} f_{i}(\boldsymbol{r}, t) \\
\rho(\boldsymbol{r}, t)=(1-\sigma(\boldsymbol{r})) \sum_{i=1}^{Q} f_{i}(\boldsymbol{r}, t) \\
\rho^{*}(\boldsymbol{r}, t) \boldsymbol{u}^{*}(\boldsymbol{r}, t)=\sum_{i=1}^{Q} f_{i}(\boldsymbol{r}, t) \boldsymbol{e}_{i} \\
\rho(\boldsymbol{r}, t) \boldsymbol{u}(\boldsymbol{r}, t)=(1-\sigma(\boldsymbol{r})) \sum_{i=1}^{Q} f_{i}(\boldsymbol{r}, t) \boldsymbol{e}_{i}
\end{gathered}
$$

where the asterix signifies a microscopic variable used for the calculation of the equilibrium functions. Equation (8) recovers eq. (6) in the case of $\sigma(\boldsymbol{r})=0$ and eq. (7) in the case of $\sigma(\boldsymbol{r})=1$. Furthermore, for $\sigma(\boldsymbol{r})=0$, equations (10) and (12) recover eq. (9) and (11), respectively while for $\sigma(\boldsymbol{r})=1$ they are both equal to zero.

Walsh et al. [24] derived the intrinsic permeability of partially-permeable homogeneous media for which $\sigma(\boldsymbol{r})=\sigma \forall \boldsymbol{r}$ as a function of the fluid kinematic viscosity and the effective media parameter:

$$
\kappa=\frac{1-\sigma}{2 \sigma} v
$$

\subsection{Implementation of the algorithm}

The partial bounce-back algorithm (equations 8 -12) was implemented in an in-house lattice Boltzmann solver written in C and parallelized using the OpenMP library. The simulations were carried out on a three-dimensional cubic lattice of size $N_{x} \times N_{y} \times N_{z}$ with nodes located at 
$\left(n_{x}, n_{y}, n_{z}\right)=(0,0,0) \ldots\left(N_{x}-1, N_{y}-1, N_{z}-1\right)$ and $Q=19$ velocities (D3Q19). All the nodes were initialized to a microscopic density according to:

$$
\rho^{*}(r, t=0)=\left\{\begin{array}{l}
0 \text { for impermeable solids } \\
1 \text { for weakly-permeable solids } \\
1 \text { for pores }
\end{array}\right.
$$

and zero microscopic velocity $\boldsymbol{u}^{*}(\boldsymbol{r}, t=0)=0$. Inlet and outlet chambers were included on the left $\left(n_{x}=0 \ldots 4\right)$ and right hand $\left(n_{x}=N_{x}-5 \ldots N_{x}-1\right)$ sides of the samples. Due to the equation of state linking the pressure $P$ to the density $\rho$ by $P=c_{s}^{2} \rho$, it is possible to drive the fluid by setting constant pressures at the inlet $\left(P=P_{\text {inlet }}, n_{x}=0\right)$ and outlet $\left(P=P_{\text {outlet }}<P_{\text {inlet }}, n_{x}=N_{x}-1\right)$ following Zou and He [39] and Narváez and Harting [40]. Periodic boundary conditions were used to minimize boundary effects in the other two directions. The pressure difference was calculated between $n_{x}=5$ and $n_{x}=N_{x}-6$ as described by Narváez et al. [41]. The permeability follows from Darcy's law (equation (1)). Convergence was considered to be achieved when the relative permeability change per 1000 time steps was less than $10^{-5}$.

\subsection{Validation of the algorithm}

In this section, we validate the implemented algorithm by comparing the numerical results with analytical predictions for a suite of standard test cases including flows in square pipes, flows through arrays of overlapping spheres, and flows through homogenous media with effective transport properties.

First, to verify that the algorithm recovered the standard lattice Boltzmann model behaviour, the flow was computed between two parallel infinite impermeable plates. The relative error in permeability of the resultant Poiseuille flow, $\varepsilon(\kappa)=\left|\kappa^{L B}-\kappa^{\text {theory }}\right| / \kappa^{\text {theory }}$, decreases from 13 to $1 \%$ when the width of the channel increases from 3 to 7 nodes.

The accuracy of the model in a more complicated geometry was tested by computing the flow through a cubic array of overlapping impermeable spheres of radius $r$ and centre-to-centre spacing 
L. The lattice resolution was varied while keeping the porosity constant at circa $20 \%$. The theoretical permeability is given by $\kappa^{\text {theory }}=\frac{1}{6 \pi C_{D}} \frac{L^{3}}{r}$ where $C_{D}$ is the porosity dependent drag force coefficient as defined in Ref. [42]. Figure 2(a) shows the simulated and theoretical permeability as well as the relative error in permeability as a function of the size of the system. There is a very good agreement. With increasing resolution, the error decreases and the calculated permeability converges to the theoretical value. For this standard validation test, the numerical error is comparable to those previously obtained using conventional finite difference schemes [43] and lattice Boltzmann solvers with single [43] and multiple [20] relaxation time collision operators. We note, however, that the error does not decrease monotonically, presumably due to the discretization of the spheres as previously observed [20, 43].

To validate the partial bounce-back algorithm, the permeability of a 10 x $10 \times 10$ lattice uniformly filled with nodes of effective media parameter $\sigma(\boldsymbol{r})=\sigma \forall \boldsymbol{r}$ was calculated as a function of $\sigma$ for a lattice spacing $\Delta x=0.5 \mu \mathrm{m}$. Figure 2(b) compares the simulation results to theory (equation (13)). The difference is smaller than $1 \%$ for homogeneous media with permeability $\kappa>10^{-19} \mathrm{~m}^{2}$ and smaller than $5 \%$ for $\kappa>10^{-23} \mathrm{~m}^{2}$. The permeability results are in excellent agreement with the corresponding two-dimensional simulations performed by Walsh et al. [24] since, for homogeneous media, the number of spatial dimensions does not matter because $\sigma(\boldsymbol{r})=\sigma \forall \boldsymbol{r}$. Indeed, it is worth noting that one of the methods that can be used to derive equation (13) starts by projecting the 3D lattice onto a 1D lattice (see Appendix A of [24]). The case of $\sigma=0.5$ is a special case and may be interpreted as a checkerboard structure with 50\% impermeable solids and 50\% pores: the permeability of a checkerboard structure equals the permeability of a homogeneous medium for which $\sigma(\boldsymbol{r})=0.5 \forall \boldsymbol{r}$. In this case, $\kappa_{\text {checkerboard }}=\kappa_{\sigma=0.5}=0.0833 \Delta x^{2}$. Additionally, the pressure drop is found to be linear inside the samples with a coefficient of determination $R^{2}>0.99$. 


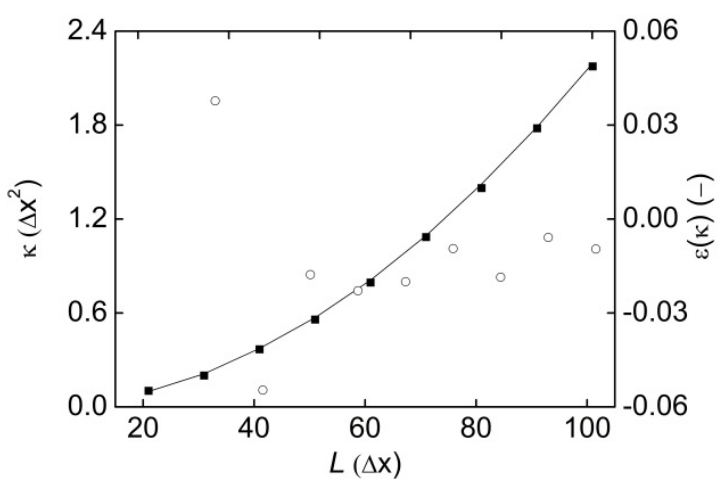

(a)

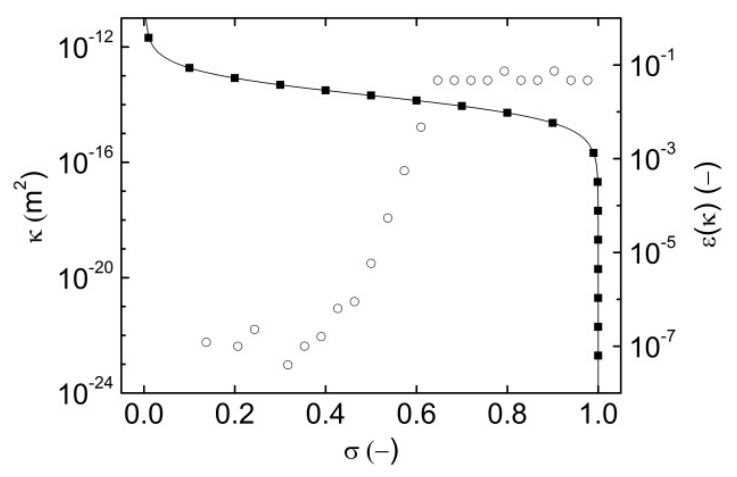

(b)

Figure 2. Analytical (solid line) and numerical (squares) permeability $\kappa$ of (a) overlapping spheres as a function of the size of the system $L$ for a constant porosity circa $20 \%$ and (b) a homogeneous system as a function of the effective media parameter $\sigma$ ranging from 0.01 to 1 $10^{-9}$ for a lattice spacing $\Delta x=0.5 \mu \mathrm{m}$. The fractional error $\varepsilon(\kappa)$ is shown with circles against the alternate axis.

In further tests, the permeability of structures built from layers of different permeability phases was calculated. Analytical expressions may be derived by analogy with the resistance of parallel and series networks of electrical resistors. In the simplified case where the structure is formed of two equal layers of permeability $\kappa_{1}$ and $\kappa_{2}$, the equivalent permeability for the parallel and series configurations is: $\kappa_{\text {parallel }}=\left(\kappa_{1}+\kappa_{2}\right) / 2$ and $\kappa_{\text {series }}=2 \kappa_{1} \kappa_{2} /\left(\kappa_{1}+\kappa_{2}\right)$. The permeability was computed while varying the permeabilities, and the ratio between the permeabilities, of the two layers. The simulations and the analytical predictions are in excellent agreement for the series configurations (error below 1\%) and in good agreement for parallel configurations (error below 10\%). In the latter case, the discrepancy is attributed to non-linearity of the pressure field. Also, the flow was computed inside a cubic sample comprising very low permeability nodes surrounding a hollow sphere. Since the flow is conserved and $\kappa_{\text {sphere }}>>\kappa_{\text {sample }}$, the pressure drop is not linear throughout the sample.

Rather, as expected, an extremely small pressure drop was observed inside the sphere. Finally, it is noted that in all tests, mass was conserved up to the numerical accuracy of the simulation. 
In all the validation tests described above, a very good agreement was observed between numerical and analytical results. This suggests that the implemented algorithm can be trusted to compute the flow in more complex geometries where analytical solutions cannot be found.

\section{Permeability of cement paste}

\subsection{Cement paste model microstructures}

Cement paste model microstructures $(100 \mu \mathrm{m})^{3}$ in size were created using the vector model $\mu$ ic [44]. The structures were generated with an initial water-to-cement $(w / c)$ ratio of 0.4 . Except when stated otherwise, the smallest and largest anhydrous particle sizes were set to 0.5 and $20 \mu \mathrm{m}$ respectively, the former corresponding to the LB lattice unit and the latter to $20 \%$ of the structure size. The phase composition of the model cement clinker was $51.9 \%$ alite, $23.1 \%$ belite, $8.1 \%$ ferrite, $9.8 \%$ aluminate, and $7.1 \%$ gypsum by solid mass. The reaction kinetics were defined according to the hydration model of Parrot and Killoh [45]. The hydrate densities were set to standard literature values

$[20,46]$ except for C-S-H that was set at $\rho_{C-S-H}=1.96 \mathrm{~g} / \mathrm{cm}^{3}$ in accordance with the recent findings of Muller et al. [27, 47]. The phase composition of the hydrated microstructure was verified and found to be in good agreement with thermodynamic calculations determined using the Gibbs Energy Minimization Software for Geochemical Modeling [48]. Microstructures were generated for different degrees of hydration and those with 9.2\% total capillary porosity (see next section) that corresponded to a degree of hydration of $89 \%$ were selected for further analysis. Except when stated otherwise, these vector based microstructures were subsequently translated onto a cubic grid of $200^{3}$ voxels with a lattice spacing of $0.5 \mu \mathrm{m}$ by taking the phase present at each voxel centre as representative of that voxel. Finally, the discretized model microstructures containing capillary pores, C-S-H, and solid inclusions were used as an input for the lattice Boltzmann simulations.

\subsection{Microstructures at reduced degrees of water saturation}


The capillary porosity of cement paste at large degrees of hydration is largely devoid of water. For example, Muller et al. [27] recently showed by ${ }^{1} \mathrm{H}$ nuclear magnetic resonance relaxation analysis that for a white cement paste with $w / c=0.4$, the total capillary porosity is $9.2 \%$ by volume after 28 days of hydration and that this comprises $1.4 \%$ of unconsumed water and $7.8 \%$ of 'empty' chemical shrinkage voids. They further showed that it is difficult to subsequently refill these voids [28]. The modelling platform $\mu$ ic generates the microstructure of the solid phases. It is usually assumed that the remaining space is filled with water. This is equivalent to ideal underwater curing. To model permeability as a function of water saturation, water was removed progressively from model microstructures, starting from the largest pores according to the Kelvin-Laplace law, as illustrated in figure 3(a-c). The largest pores were determined using a porosimetry algorithm developed by Do et al. [49]. To avoid systematic bias due to the pore-emptying algorithm, multiple repeats of the algorithm were run, in which the order that pores of any given size were emptied was randomly chosen. This process yielded multiple partially saturated structures with the same saturated pore size distribution originating from a given saturated parent structure. The process was repeated for different parent structures all with the same overall capillary porosity (9.2\%). The emptied pores were considered impermeable to liquid water, along with the solid phases.

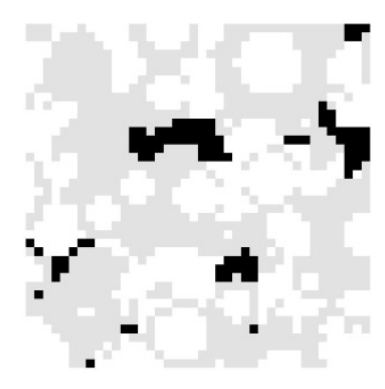

(a)

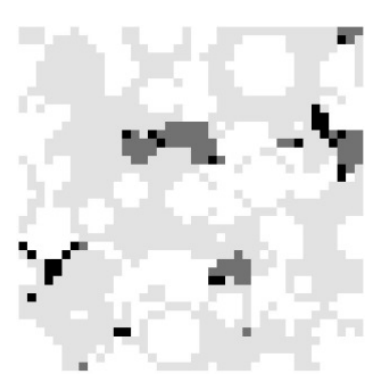

(b)

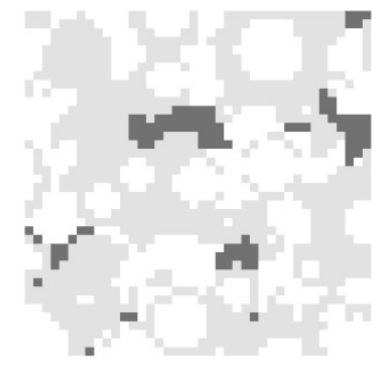

(c)

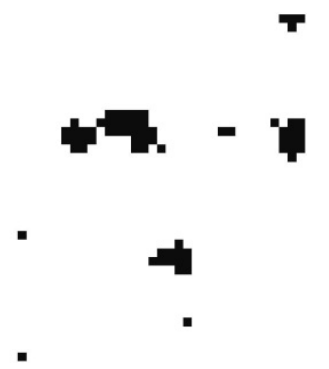

(d)

Figure 3. A $40^{2}$ pixels slice of a $200^{3}$ voxels $\mu$ ic cement paste microstructure at different degrees of water saturation. In $(a-c)$, solid impermeable phases are shown in white, weakly-permeable C-S-H in light grey, emptied capillary pores in dark grey and water-filled capillary pores in black. In (a) the capillary porosity is fully saturated with water; in (b) it is partially saturated and in (c) it is empty of water and fully saturated with gas. (d) Shows the sister structure of (b) 
created when the permeating fluid is gas. In (d) the solids, water and C-S-H are considered impermeable and appear white while the permeable void spaces are black.

Powers gave the coefficient of water permeability of the C-S-H gel as $7 \times 10^{-23} \mathrm{~m}^{2}$ [50]. This permeability was assigned to all the C-S-H nodes through application of equation (13) so that $\sigma_{C-S-H}=1-3.36 \times 10^{-9}$ when the lattice spacing $\Delta x$ is equal to $0.5 \mu \mathrm{m}$. As this is the only value that could be found in literature, the dependency of the bulk permeability on the C-S-H permeability was investigated by increasing and decreasing the latter by a factor of 10 .

In order to compare liquid and gas permeability, sister structures were generated in which the C-S-H was considered impermeable to gas and the "LB fluid" and "LB solid” parts of the partially saturated capillary porosity were systematically reversed as shown in figures 3(b) and 3(d). These modifications enabled the use of a single-phase LB model to study the gas permeability. Table II summarizes the setup of the simulations.

Table II: Water and gas permeability of the different cement paste phases.

\begin{tabular}{lllll}
\hline $\begin{array}{l}\text { Permeating } \\
\text { fluid }\end{array}$ & Solid phases & $\begin{array}{l}\text { Water-filled } \\
\text { capillary pores }\end{array}$ & $\begin{array}{l}\text { Empty } \\
\text { capillary pores }\end{array}$ & $\kappa_{\mathrm{C}-\mathrm{s}-\mathrm{H}}\left(10^{-23} \mathrm{~m}^{2}\right)$ \\
\hline Water & Impermeable & Solve Navier-Stokes & Impermeable & 70,7 and 0.7 \\
Gas & Impermeable & Impermeable & Solve Navier-Stokes & Impermeable \\
\hline
\end{tabular}

\subsection{Apparent intrinsic permeability of cement paste model microstructures}

Figure 4 (left) shows the cement paste apparent intrinsic permeability with water as the permeating fluid as a function of the degree of water saturation of the capillary porosity for $\kappa_{C-S-H}=7 \times 10^{-23} \mathrm{~m}^{2}$. The graph also shows the gas permeability for the sister structures, in which the fluid-accessible pore space comprises the larger emptied capillary pores and the C-S-H is impermeable, mimicking a gas permeability experiment. Figure 4 (right) shows selected experimental cement paste permeabilities taken from table I and the references therein. 

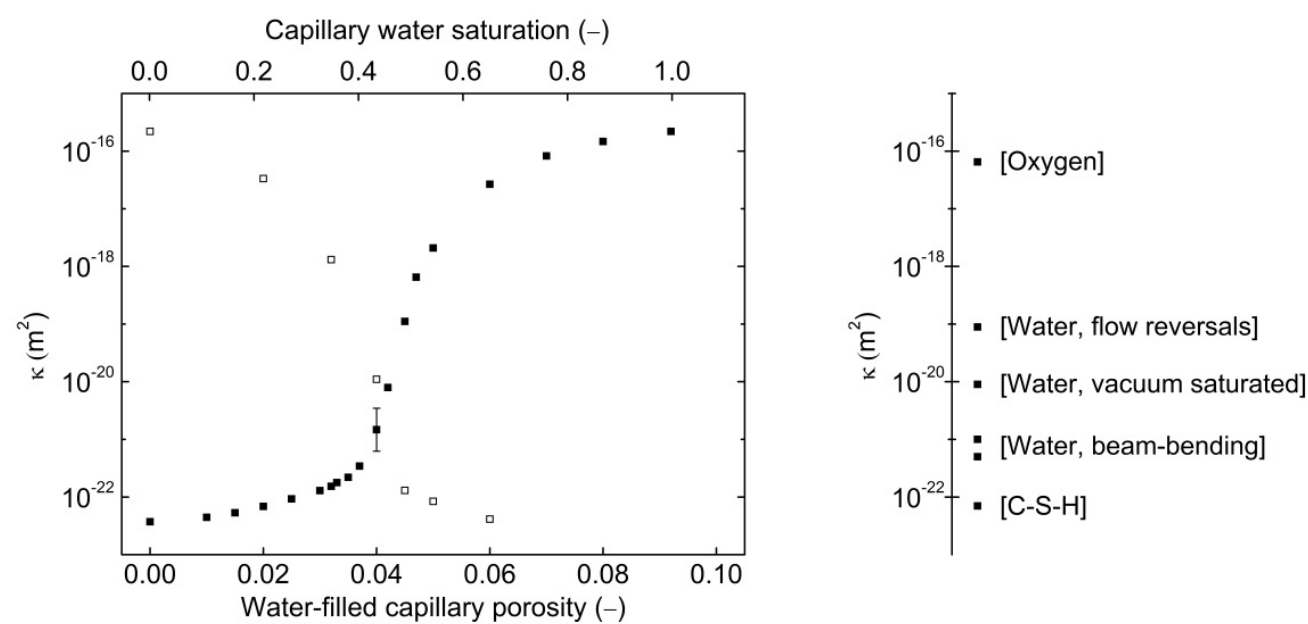

Figure 4. (left) Simulated apparent intrinsic permeability $\kappa$ as a function of the degree of water saturation. The penetrating fluid is water (full squares) or gas (open squares). The model cement paste water-to-cement ratio is 0.4 and the capillary porosity corresponding to full saturation is $9.2 \%$. An exemplar error bar (mean \pm standard deviation) is shown close to the depercolation threshold. Away from the depercolation threshold, water-filled porosity less than 0.035 or greater than 0.05, the error bar is smaller than the symbol size. (right) Experimental cement paste permeabilities from table I, from top to bottom: Wong et al. [13], Banthia \& Mindess [9], Ye [12], Vichit-Vadakan \& Scherer [4, 7], Vichit-Vadakan \& Scherer [4, 7] and Powers [50].

Close to $50 \%$ water saturation, the convergence criterion, as defined in section 3.3 , was not always reached. In these cases, the simulations were stopped after one million time steps and the relative change in permeability per 1000 time steps was typically $10^{-4}$. The standard deviation of the water permeability has been calculated from multiple runs of the simulation. It comprises two parts: the first is variability in the parent structure (initial anhydrous cement particles positions); the second is variability in the water distribution within a given structure. The variation is negligible when the structures are highly saturated with a fluid and highest near the depercolation threshold. An exemplar standard deviation is shown on figure 4 (left) near the depercolation threshold for a confidence interval of $68.2 \%$ (mean \pm standard deviation). The distribution width of the simulated water permeability of nominally similar samples is comparable to what is observed experimentally (table I). 
To test the effect of microstructural resolution on permeability, further structures were generated with a smaller minimum anhydrous cement particle size and LB lattice spacing of $0.25 \mu \mathrm{m}$. By increasing the resolution, the structural complexity of the model cement paste increases. At full water saturation, the water permeability decreases by circa 3.5 times, in agreement with previous observations [19, 20]. However, compared to the six orders of magnitude spanned by the data, this now seems a small factor. Moreover, the water permeability does not change with resolution for structures with no water-filled capillary porosity.

To test the effect of the C-S-H permeability on the water permeability of the paste, simulations were re-run using $\kappa_{C-S-H}=7 \times 10^{-22} \mathrm{~m}^{2}$ and $\kappa_{C-S-H}=7 \times 10^{-24} \mathrm{~m}^{2}$. The results are shown in Figure 5. As expected, changing the C-S-H permeability has no effect on the water permeability for structures with high water saturation since the water-filled capillary porosity is percolated and $\kappa_{C-S-H} \ll \kappa_{\text {paste }}$. The effect of the C-S-H permeability on the water permeability of the paste amplifies with decreasing water saturation. At 1.5\% water-filled capillary porosity, increasing the C-S-H permeability by a factor of 10 increases the water permeability by a factor $\approx 7$, while decreasing the C-S-H permeability by 10 decreases the water permeability by $\approx 3$ times only. At zero water-saturation, the effect of the C-S-H permeability on the paste permeability becomes linear so that the modelled water permeabilities are $3.7 \times 10^{-22} \mathrm{~m}^{2}, 3.7 \times 10^{-23} \mathrm{~m}^{2}$ and $3.7 \times 10^{-24} \mathrm{~m}^{2}$ for structures with C-S-H permeabilities of $7 \times 10^{-22} \mathrm{~m}^{2}, 7 \times 10^{-23} \mathrm{~m}^{2}$ and $7 \times 10^{-24} \mathrm{~m}^{2}$ respectively. These values are consistent with the idea that the C-S-H occupies approximately half of the volume of the cement paste. 


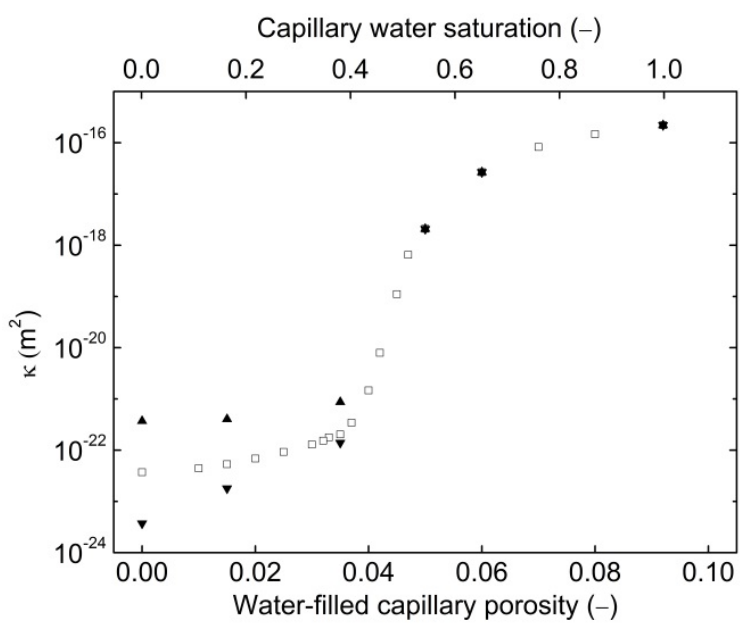

Figure 5. Simulated apparent intrinsic permeability $\boldsymbol{\kappa}$ as a function of the degree of water saturation for different values of the C-S-H permeability. The penetrating fluid is water. The CS-H permeability is $7 \times 10^{-22} \mathrm{~m}^{2}$ (up triangles), $7 \times 10^{-23} \mathrm{~m}^{2}[50]$ (open squares) or $7 \times 10^{-24} \mathrm{~m}^{2}$ (down triangles). The model cement paste water-to-cement ratio is 0.4 and the capillary porosity corresponding to full saturation is $\mathbf{9 . 2 \%}$. The variation of the C-S-H permeability is made to give an indication of how this impacts the overall paste permeability. Simulations were only run at a limited number of saturation levels for reasons of time.

\section{Discussion}

The intrinsic permeability of the structure fully saturated with water is conceptually the same as the intrinsic permeability of a completely dried cement paste saturated with gas in an ideal gas permeability experiment. It is therefore not surprising that, at high degrees of water saturation, the water permeability is comparable to the oxygen permeability results of Wong et al. [13]. Practically, a better comparison is with the simulation of the sister structure saturated with gas (zero water saturation) since, in most laboratory gas experiments, the C-S-H is not fully dried and is therefore impermeable to gas. In both cases, when the capillary porosity is highly saturated with a fluid, the role of water within the C-S-H in determining permeability is minimal as the flow is mainly controlled by the percolated fluid-filled capillary pores. 
By gradually reducing the water saturation, the intrinsic water permeability decreases. Initially the decrease is slow. Below $60 \%$ water saturation, the water permeability decreases more rapidly as the water-filled capillary porosity starts to depercolate. We observe a very sharp drop in water permeability between 50 and $40 \%$ water saturation regardless of the C-S-H permeability.

When the water-filled capillary porosity is depercolated, the influence of flow through the weakly-permeable C-S-H is amplified because the flow is then mainly controlled by the weaklypermeable C-S-H. The water permeability ranges from approximately $10^{-19}$ to $10^{-22} \mathrm{~m}^{2}$. This agrees well with the range of experimental water permeability measurements (table I). It is therefore interesting to consider the likely degree of water saturation of the samples studied. In the case of Ye [12], paste samples were sealed cured for 28 days and then vacuum saturated with water for 4 to 8 hours before the water permeability measurement. Muller [28] reports that for a 28 day sealed cured sample that was subsequently vacuum saturated for 24 hours, the water-filled capillary porosity rose from 1.4 to $5.5 \%$ as measured by NMR. Since Ye’s samples were vacuum saturated for a shorter time, a slightly smaller water-filled fraction should be expected. In agreement with this expectation, the water permeability measurement of Ye, $9.0 \times 10^{-21} \mathrm{~m}^{2}$, is matched by a water permeability simulation with approximately $4.3 \%$ water-filled capillary porosity for Powers measured value $\kappa_{C-S-H}=7 \times 10^{-23} \mathrm{~m}^{2}$.

Vichit-Vadakan and Scherer [4] used the beam-bending method to measure water permeability in a range of samples of different sizes that were cured sealed for 48 hours and then further cured underwater. Permeability is derived from the rate of relief of pressure required to bend a water saturated sample. To verify that the samples were fully saturated, they compared the permeability before and after pressurization underwater at 2 MPa for 24 hours. The authors concluded that small samples were fully saturated prior to pressurization on the basis of constancy of the permeability but that this was not the case for larger samples. However, a careful examination of their figure 4 data reveals that permeability decreased after pressurization by about $20 \%$ for the smallest samples $(5.7 \mathrm{~mm})$ and by about $60 \%$ for slightly larger samples $(7.7 \mathrm{~mm})$. As they suggest, one possible interpretation for higher permeability values before pressure saturation is the relief of internal 
pressure by water flow into nearby air voids. We suggest that even the small samples may not have been fully saturated before pressurization. Moreover, those few samples that were subjected to pressurization may not have been fully saturated either. Using the experimentally measured permeability and a pressure of $2 \mathrm{MPa}$, Darcy's law suggests that several days are required to fill the void spaces created during the preliminary 48 hours of sealed curing, rather than the single day allowed, even for the small samples. In the LB simulations, the water permeability matches the experimental measurements of Vichit-Vadakan and Scherer $\left(5 \times 10^{-22}\right.$ to $\left.10^{-21} \mathrm{~m}^{2}\right)$ between 4 and $4.2 \%$ water-filled capillary porosity again for $\kappa_{C-S-H}=7 \times 10^{-23} \mathrm{~m}^{2}$ as reported by Powers.

Overall, the effect of the C-S-H on the simulated permeability is partially seen in the detail of the asymmetry of the "liquid" and "gas" curves in figure 4 (left). This is because the C-S-H is considered permeable in the former case, but is not in the latter. However, it is not the full story, since the asymmetry is also due to the fact that at water saturation $S_{w}$, the liquid occupies a different pore network (predominantly small pores) compared to the gas (large pores) at saturation $1-S_{w}$. The role of the C-S-H amplifies as the capillary porosity starts to depercolate. This is unequivocally seen by comparing the current results with previous LB water permeability simulations that treated the C-S-H as impermeable solid [19-21]. In those calculations, as the porosity decreased (by increasing the degree of hydration) the permeability decreased monotonically and catastrophically close the percolation threshold, below which it could not be calculated accurately. Now, however, the asymptotic approach towards a plateau permeability dictated by the C-S-H is clearly seen. As a final comment, we note that while we have looked at both liquid water and gas permeability, we have not yet considered a multi-phase fluid with an equation of state that links liquid water and vapour. Such analyses have been attempted within analytic models [18] and their exploration will be the subject of future numerical simulations.

\section{Conclusions}

This work has shown that a 3D effective media lattice Boltzmann approach is well suited to numerical calculation of permeability within complex multi-scale 3D cement paste microstructures 
including capillary pore regions of defined geometry, calcium silicate hydrate regions of defined permeability, and impermeable solid inclusions. This is an advance over previous numerical simulations of permeability in cement paste where only the capillary pore network was considered, and further this was fully saturated. The simulation of apparent intrinsic permeability at different degrees of water saturation provides a very plausible explanation for the enormous range of experimental results. The results also explain the apparently large values of water permeability reported in previous simulations without invoking earlier arguments of limited spatial resolution either in the microstructure or in the lattice Boltzmann solver. It is now clear that the large permeability is a direct consequence of the percolation of the capillary pore network at full water saturation. The suggestion is that most experimental measurements with water are not conducted on fully saturated samples. The presence of air voids in nominally water saturated samples dramatically lowers their apparent intrinsic permeability.

\section{Acknowledgements}

Funding was provided by the European Union (FP7/2007-2013 grant 264448) and the UK EPSRC (grant EP/H033343/1). We thank Aslam Kunhi Mohamed and Quang Huy Do (both Ecole Polytechnique Fédérale de Lausanne, Switzerland) for helping with the generation of the $\boldsymbol{\mu i c}$ model microstructures and the calculation of the pore size distributions.

\section{References}

[1] E. Gartner, Industrially interesting approaches to “low-CO2” cements, Cem. Conc. Res. 34 (2004) 1489-1498.

[2] World Business Council for Sustainable Development, Cement Sustainability Initiative, http://www.wbcsdcement.org, (accessed $15^{\text {th }}$ March 2013).

[3] H. Darcy, Les Fontaines Publiques de la Ville de Dijon, Dalmont, Paris, 1856.

[4] W. Vichit-Vadakan and G.W. Scherer, Measuring Permeability of Rigid Materials by a BeamBending Method: III, Cement Paste, J. Am. Ceram. Soc. 85 (2002) 1537-1544. 
[5] P.B. Bamforth, The Relationship between Permeability Coefficients for Concrete Obtained Using Liquid and Gas, Mag. Concrete Res. 39 (1987) 3-11.

[6] H. Loosveldt, Z. Lafhaj and F. Skoczylas, Experimental study of gas and liquid permeability of a mortar, Cem. Conc. Res. 32 (2002) 1357-1363.

[7] Z.C. Grasley, G.W. Scherer, D.A. Lange and J.J. Valenza, Dynamic pressurization method for measuring permeability and modulus: II. cementitious materials, Mater. Struct. 40 (2007) 711-721. [8] B.K. Nyame and J.M. Illston, Relationships between permeability and pore structure of hardened cement paste, Mag. Concrete Res. 33 (1981) 139-146.

[9] N. Banthia and S. Mindess, Water permeability of cement paste, Cem. Conc. Res. 19 (1989) 727736.

[10] H. Ai, J.F. Young and G.W. Scherer, Thermal Expansion Kinetics: Method to Measure Permeability of Cementitious Materials: II, Application to Hardened Cement Pastes, J. Am. Ceram. Soc. 84 (2001) 385-391.

[11] W. Vichit-Vadakan and G.W. Scherer, Measuring permeability and stress relaxation of young cement paste by beam bending, Cem. Conc. Res. 33 (2003) 1925-1932.

[12] G. Ye, Percolation of capillary pores in hardening cement pastes, Cem. Conc. Res. 35 (2005) $167-176$.

[13] H.S. Wong, M. Zobel, N.R. Buenfeld and R.W. Zimmerman, Influence of the interfacial transition zone and microcracking on the diffusivity, permeability and sorptivity of cement-based materials after drying, Mag. Concrete Res. 61 (2009) 571 -589.

[14] R.K. Dhir, P.C. Hewlett and Y.N. Chan, Near surface characteristics of concrete: intrinsic permeability, Mag. Concrete Res. 41 (1989) 87-97.

[15] N. Hearn, R.J. Detwiler and C. Sframeli, Water permeability and microstructure of three old concretes, Cem. Conc. Res. 24 (1994) 633-640.

[16] A. Abbas, M. Carcasses and J.P. Ollivier, Gas permeability of concrete in relation to its degree of saturation, Mater. Struct. 32 (1999) 3-8.

[17] H. Wong, N. Buenfeld, J. Hill and A. Harris, Mass transport properties of mature wasteform grouts, Adv. Cem. Res. 19 (2007) 35-46. 
[18] V. Baroghel-Bouny, M. Thiéry and X. Wang, Modelling of isothermal coupled moisture-ion transport in cementitious materials, Cem. Conc. Res. 41 (2011) 828-841.

[19] E.J. Garboczi and D.P. Bentz, The effect of statistical fluctuation, finite size error, and digital resolution on the phase percolation and transport properties of the NIST cement hydration model, Cem. Conc. Res. 31 (2001) 1501-1514.

[20] M. Zalzale and P.J. McDonald, Lattice Boltzmann simulations of the permeability and capillary adsorption of cement model microstructures, Cem. Conc. Res. 42 (2012) 1601-1610.

[21] M. Zhang, G. Ye and K.v. Breugel, Microstructure-based modeling of permeability of cementitious materials using multiple-relaxation-time lattice Boltzmann method, Comp. Mater. Sci. 68 (2013) 142-151.

[22] O. Dardis and J. McCloskey, Lattice Boltzmann scheme with real numbered solid density for the simulation of flow in porous media, Phys. Rev. E 57 (1998) 4834-4837.

[23] D. Thorne and M. Sukop, Lattice Boltzmann model for the Elder problem, and Proceedings in XVth International Conference on Computational Methods in Water Resources (CMWR XV), Chapel Hill, NC, USA, 2004

[24] S.D.C. Walsh, H. Burwinkle and M.O. Saar, A new partial-bounceback lattice-Boltzmann method for fluid flow through heterogeneous media, Comput. Geosci. 35 (2009) 1186-1193. [25] R. Feldman and P. Sereda, A new model for hydrated Portland cement and its practical implications, Engineering Journal 53 (1970) 53-59.

[26] H.M. Jennings, Refinements to colloid model of C-S-H in cement: CM-II, Cem. Conc. Res. 38 (2008) 275-289.

[27] A.C.A. Muller, K.L. Scrivener, A.M. Gajewicz and P.J. McDonald, Densification of C-S-H measured by 1H NMR relaxometry, J. Phys. Chem. C 117 (2013) 403-412.

[28] A.C.A. Muller, Vacuum saturation of the air voids inside sealed cured cement samples: a $1 \mathrm{H}$ nuclear magnetic resonance relaxation analysis, Personal Communication, 2012.

[29] H. Chen, S. Chen and W.H. Matthaeus, Recovery of the Navier-Stokes equations using a latticegas Boltzmann method, Phys. Rev. A 45 (1992) 5339-5342. 
[30] X. He, Q. Zou, L.-S. Luo and M. Dembo, Analytic solutions of simple flows and analysis of nonslip boundary conditions for the lattice Boltzmann BGK model, J. Stat. Phys. 87 (1997) 115-136. [31] M.A.A. Spaid and J.F.R. Phelan, Lattice Boltzmann methods for modeling microscale flow in fibrous porous media, Phys. Fluids 9 (1997) 2468-2474.

[32] D.M. Freed, Lattice-Boltzmann Method for Macroscopic Porous Media Modeling, Int. J. Mod. Phys. C 09 (1998) 1491-1503.

[33] N.S. Martys, Improved approximation of the Brinkman equation using a lattice Boltzmann method, Phys. Fluids 13 (2001) 1807-1810.

[34] N.S. Martys and J.G. Hagedorn, Multiscale modeling of fluid transport in heterogeneous materials using discrete Boltzmann methods, Mater. Struct. 35 (2002) 650-658.

[35] O. Dardis and J. McCloskey, Permeability porosity relationships from numerical simulations of fluid flow, Geophys. Res. Lett. 25 (1998) 1471-1474.

[36] K. Balasubramanian, F. Hayot and W.F. Saam, Darcy’s law from lattice-gas hydrodynamics, Phys. Rev. A 36 (1987) 2248-2253.

[37] Y. Gao and M.M. Sharma, A LGA model for fluid flow in heterogeneous porous media, Transport Porous Med. 17 (1994) 1-17.

[38] M. Sukop and D. Thorne, Lattice Boltzmann Modeling: An Introduction for Geoscientists and Engineers, Springer, 2006.

[39] Q. Zou and X. He, On pressure and velocity boundary conditions for the lattice Boltzmann BGK model, Phys. Fluids 9 (1997) 1591-1598.

[40] A. Narváez and J. Harting, Evaluation of pressure boundary conditions for permeability calculations using the lattice-Boltzmann method, Adv. Appl. Math. Mech. 2 (2010) 685-700. [41] A. Narváez, T. Zauner, F. Raischel, R. Hilfer and J. Harting, Quantitative analysis of numerical estimates for the permeability of porous media from lattice-Boltzmann simulations, J. Stat. MechTheory E. 11 (2010) P11026.

[42] R.E. Larson and J.J.L. Higdon, A periodic grain consolidation model of porous media, Phys. Fluids A 1 (1989) 38-46. 
[43] C. Manwart, U. Aaltosalmi, A. Koponen, R. Hilfer and J. Timonen, Lattice-Boltzmann and finite-difference simulations for the permeability for three-dimensional porous media, Phys. Rev. E 66 (2002) 016702.

[44] S. Bishnoi and K.L. Scrivener, $\mu$ ic: A new platform for modelling the hydration of cements, Cem. Conc. Res. 39 (2009) 266-274.

[45] L.J. Parrot and D.C. Killoh, Prediction of cement hydration, Proc. Br. Ceram. Soc. 35 (1984) 4153.

[46] H.F.W. Taylor, Cement chemistry, second ed., Thomas Telford, London, 1997.

[47] A.C.A. Muller, K.L. Scrivener, A.M. Gajewicz and P.J. McDonald, Use of bench-top NMR to measure the density, composition and desorption isotherm of $\mathrm{C}-\mathrm{S}-\mathrm{H}$ in cement paste, Micropor. Mesopor. Mat. 178 (2013) 99-103.

[48] GEMS, http://gems.web.psi.ch/, (accessed $15^{\text {th }}$ March 2013).

[49] Q.H. Do, S. Bishnoi and K.L. Scrivener, Numerical Simulation of Porosity in Cements, Transport Porous Med. 99 (2013) 101-117.

[50] T.C. Powers, Structure and Physical Properties of Hardened Portland Cement Paste, J. Am. Ceram. Soc. 41 (1958) 1-6. 